\title{
Study of theoretical luminosity precision for electron colliders at higher energies
}

\author{
S. Jadach ${ }^{1}$, W. Płaczek ${ }^{2}$, M. Skrzypek ${ }^{1, \mathrm{a}}{ }^{\oplus}$, B. F. L. Ward $^{3}$ \\ ${ }^{1}$ Institute of Nuclear Physics, Polish Academy of Sciences, ul. Radzikowskiego 152, 31-342 Kraków, Poland \\ ${ }^{2}$ Institute of Applied Computer Science, Jagiellonian University, ul. Łojasiewicza 11, 30-348 Kraków, Poland \\ ${ }^{3}$ Baylor University, Waco, TX, USA
}

Received: 20 September 2021 / Accepted: 21 November 2021 / Published online: 30 November 2021

(C) The Author(s) 2021

\begin{abstract}
We present an estimation of the theoretical precision of low angle Bhabha scattering at the proposed future ILC collider at $500 \mathrm{GeV}$. The analysis is an extension of the previous analysis done for the FCCee collider at $\sqrt{s}=M_{Z}$. As the state-of-the-art and the reference point we use the BHLUMI Monte Carlo event generator. Based on the current precision status of BHLUMI for LEP analysis, we estimate how various error components evolve from the LEP to ILC setups. The conclusion of our work is that for the ILC the precision of the current version of BHLUMI 4.04 deteriorates to $0.5 \%$, by more than an order of magnitude w.r.t. the present precision for LEP. With the expected future improvements, the precision of BHLUMI can change to $0.016 \%$, nearly as good as for the FCCee at the $M_{Z}$ setup $(0.01 \%)$. Based on the developed methodology we present also results for ILC $\mathrm{I}_{1000}$, FCCee $_{350}$ and $\mathrm{CLIC}_{3000}$ setups.
\end{abstract}

\section{Introduction}

Next generation of the proposed future particle colliders are the $e^{+} e^{-}$machines. As in the past, the strongest point of these projects is high precision. Amongst all measurements a special position is given to the luminosity measurement. Precision of all cross-section-based observables depends on an exact knowledge of the luminosity. The preferred method of luminosity measurement is via the low angle Bhabha (LABH) scattering $e^{+} e^{-} \rightarrow e^{+} e^{-}$. On the theoretical side this process is known to depend almost only on QED inter-

This work is partly supported by the funding from the European Union's Horizon 2020 research and innovation programme under Grant agreement no. 951754 and from the National Science Centre, Poland, Grant no. 2019/34/E/ST2/00457.

a e-mail: maciej.skrzypek@ifj.edu.pl (corresponding author) actions, thus minimizing the higher order EW and QCD corrections.

In the previous paper [1] we scrutinized the LEP legacy and the current state-of-the-art situation concerning the theoretical precision of $\mathrm{LABH}$ calculations as implemented in the BHLUMI [2,3] Monte Carlo code. Based on this background we analysed the future prospects of theoretical precision of luminosity measurement at the FCCee collider at the $Z$ peak. By analysing various components of the higher order corrections we came to the conclusion that the desired relative precision of $1 \times 10^{-4}$ is feasible.

In this paper we extend further the analysis of [1] to another future $e^{+} e^{-}$collider project, the ILC. Technical parameters of the ILC and its detector differ from FCCee, but the essence of the measurement remains the same. In the following we will discuss all the corrections to $\mathrm{LABH}$ scattering for the ILC setup. We will demonstrate, that the current precision of BHLUMI is poor, at the level of $0.5 \%$, but the future prospects of $1.6 \times 10^{-4}$ are much better, only slightly worse than for FCCee.

In the next section we present the very basics of Bhabha scattering in low angles and then we discuss the setup of various colliders and current status of BHLUMI. In the next five sections we analyse various components of the error in LABH, with an eye on measurements at ILC. The Summary with additional results for ILC $_{1000}, \mathrm{FCCee}_{350}$ and $\mathrm{CLIC}_{3000}$ closes the article.

\section{Preliminary remarks}

The LABH cross section in Born approximation and in the limit of small angles is given by the formula

$\sigma_{B h} \simeq 4 \pi \alpha^{2}\left(\frac{1}{t_{\min }}-\frac{1}{t_{\max }}\right)$ 
Table 1 Basic parameters of the detectors at $e^{+} e^{-}$past and future machines. The $\theta_{\min }-\theta_{\max }$ are the generic $\mathrm{LABH}$ detector acceptances. The $\bar{t}$ is defined in Eq. (3)

\begin{tabular}{lllll}
\hline Machine & $\theta_{\min }-\theta_{\max }(\mathrm{mrad})$ & $\sqrt{s}(\mathrm{GeV})$ & $\bar{t} / s$ & $\sqrt{\bar{t}}(\mathrm{GeV})$ \\
\hline LEP & $28-50$ & $M_{Z}$ & $3.5 \times 10^{-4}$ & 1.70 \\
FCCee & $64-86$ & $M_{Z}$ & $13.7 \times 10^{-4}$ & 3.37 \\
FCCee & $64-86$ & 350 & $13.7 \times 10^{-4}$ & 13.0 \\
ILC & $31-77$ & 500 & $6.0 \times 10^{-4}$ & 12.2 \\
ILC & $31-77$ & 1000 & $6.0 \times 10^{-4}$ & 24.4 \\
CLIC & $39-134$ & 3000 & $13.0 \times 10^{-4}$ & 108 \\
\hline
\end{tabular}

For small angles the transfer $t$ can be expanded in the scattering angle $\theta$

$t \simeq-s \frac{\theta^{2}}{4}$

and the total cross section (1) can be rewritten as

$\sigma_{B h} \simeq \frac{16 \pi \alpha^{2}}{s}\left(\frac{1}{\theta_{\min }^{2}}-\frac{1}{\theta_{\max }^{2}}\right)$

$\theta_{\min / \max }^{2}$ are the angular ranges of the detector and $t_{\min / \max }$ are the corresponding transfers. Yet another form of the cross section (1) can be obtained by introducing the average transfer $\bar{t}$ defined as geometric mean

$\bar{t}=\sqrt{t_{\min } t_{\max }}$.

With the help of $\bar{t}$ one can rewrite (1) as

$\sigma_{B h} \simeq 4 \pi \alpha^{2}\left(\frac{t_{\max }-t_{\min }}{\bar{t}^{2}}\right)$,

showing the privileged role of $\bar{t}$. It turns out [1] that the $\bar{t}$ is a good scale of the LABH process used in the discussions of higher order corrections.

Another important parameter is the ratio $\bar{t} / s$ which is the suppression factor between $s$ - and $t$-channel contributions. In the Table 1 we collect these quantities for generic setups of the LEP (SiCal), FCCee [4], ILC [5] and CLIC [6] projects.

As one can see from the Table 1 the average transfer (energy scale) grows about four times from FCCee at $M_{Z}$ to ILC at $500 \mathrm{GeV}$, whereas the planned angular range $(\bar{t} / s)$ drops by factor of two. We will now discuss consequences of these changes for various subcomponents of the $\mathrm{LABH}$ error. Let us note that, as in the previous study [1] we use the BHLUMI code as the reference point in the analysis. It differs in some places from the state of the art non-MC calculations. In the Table 2, based on Refs. [1,7] we present various components of the BHLUMI error budget as of 2019 for the LEP SiCal-type setup.
Table 2 Summary of the total (physical+technical) theoretical uncertainty for a typical calorimetric LEP luminosity detector within the generic angular range of $18-52 \mathrm{mrad}$. Total error is summed in quadrature

\begin{tabular}{ll}
\hline Type of correction/error & Update 2019 \\
\hline (a) Photonic $\mathcal{O}\left(L_{e} \alpha^{2}\right)$ & $0.027 \%[8]$ \\
(b) Photonic $\mathcal{O}\left(L_{e}^{3} \alpha^{3}\right)$ & $0.015 \%[9]$ \\
(c) Vacuum polariz. & $0.009 \%[7,10]$ \\
(d) Light pairs & $0.010 \%[7]$ \\
(e) $Z$ and $s$-channel $\gamma$ exchange & $0.015 \%[11,12]$ \\
(f) Up-down interference & $0.0014 \%[13]$ \\
(g) Technical Precision & $(0.027) \%$ \\
Total & $0.037 \%$ \\
\hline
\end{tabular}

\section{BHLUMI error components}

In the following sections we will briefly discuss the origin and current status of all errors in BHLUMI and reanalyse them for the ILC setup.

\subsection{Photonic higher orders}

In BHLUMI photonic corrections are included to $\mathcal{O}(\alpha$, $\left.\alpha^{2} \ln ^{2}\left(|t| / m_{e}^{2}\right)\right)$ exponentiated. The term "exponentiated" refers to the method of the YFS [14] resummation of soft photons. That work was transformed in a pioneering work [15] into a Monte Carlo scheme used as a basis of a whole family of MC codes, BHLUMI amongst them. Contrary to leadinglog based philosophy, YFS approach guarantees correct, fully exclusive, soft limit behavior to all orders and provides an exact method of including non-soft remainders (so-called $\beta$ functions) to a given finite order. On the other hand, the YFS resummation is not exact in the leading-log limit which must be corrected perturbatively in $\beta$-functions.

Some of the missing corrections are known in the literature: $\mathcal{O}\left(\alpha^{2} \ln \left(|t| / m_{e}^{2}\right)\right)$ [16-19] and $\mathcal{O}\left(\alpha^{3} \ln ^{3}\left(|t| / m_{e}^{2}\right)\right)$ [9, 20]. These corrections will be added to BHLUMI so we do not include them into the error budget. The $\mathcal{O}\left(\alpha^{2} \ln ^{0}\left(|t| / m_{e}^{2}\right)\right)$ corrections are also known [21-28]. They are of the order of $10^{-5}$ and after some checking will most likely not be 
needed in BHLUMI. The truly missing terms are non-leading $\mathcal{O}\left(\alpha^{3} \ln ^{2}\left(|t| / m_{e}^{2}\right)\right)$ and leading $\mathcal{O}\left(\alpha^{4} \ln ^{4}\left(|t| / m_{e}^{2}\right)\right)$. We can estimate them as follows.

At LEP the $\mathcal{O}\left(\alpha^{4} \ln ^{4}\left(\left|t_{L E P}\right| / m_{e}^{2}\right)\right)$ corrections were estimated based on the size of known $\mathcal{O}\left(\alpha^{3} \ln ^{3}\left(\left|t_{L E P}\right| / m_{e}^{2}\right)\right)$ corrections. The latter ones are of the size of $1.5 \times 10^{-4}$ at LEP [9, 20]. Multiplied by the generic $(\alpha / \pi) \ln \left(\bar{t}_{L E P} / m_{e}^{2}\right)=0.039$ it gave an estimate of $6 \times 10^{-6}$ for the $\mathcal{O}\left(\alpha^{4} \ln ^{4}\left(\left|t_{L E P}\right| / m_{e}^{2}\right)\right)$ terms.

At ILC $(500 \mathrm{GeV})$ we can assume that the $\mathcal{O}\left(\alpha^{3} \ln ^{3}\right.$ $\left.\left(|t| / m_{e}^{2}\right)\right)$ term grows by a factor of $\ln ^{3}\left(\bar{t}_{I L C} / m_{e}^{2}\right) / \ln ^{3}\left(\bar{t}_{L E P} /\right.$ $\left.m_{e}^{2}\right)=1.9$, i.e. it increases to $0.028 \%$. Including an additional factor of $(\alpha / \pi) \ln \left(\bar{t}_{I L C} / m_{e}^{2}\right)=0.049$ and a safety factor of 2 we end up with the estimate of the missing term $\mathcal{O}\left(\alpha^{4} \ln ^{4}\left(\left|t_{I L C}\right| / m_{e}^{2}\right)\right) \sim 0.27 \times 10^{-4}$.

The known, but not included in BHLUMI terms $\mathcal{O}\left(\alpha^{2}\right.$ $\left.\ln \left(|t| / m_{e}^{2}\right)\right)$ were estimated at LEP to be $0.027 \%$. This is a conservative estimate because it includes also the technical precision of the whole BHLUMI program. Scaled by change of logarithm, at ILC we expect them to be of the order of $0.033 \%$. The $\mathcal{O}\left(\alpha^{3} \ln ^{2}\left(|t| / m_{e}^{2}\right)\right)$ terms are more difficult to estimate. For LEP we estimated their order of magnitude in [1] as $(\alpha / \pi)^{3} \ln ^{2}\left(\bar{t}_{L E P} / m_{e}^{2}\right) \sim 0.04 \times 10^{-4}$. At ILC we find in analogy a similar estimate $(\alpha / \pi)^{3} \ln ^{2}\left(\bar{t}_{I L C} / m_{e}^{2}\right) \sim$ $0.06 \times 10^{-4}$. Including a safety factor of 2 the estimate for ILC $_{500}$ becomes $0.12 \times 10^{-4}$. Alternatively, we can estimate that correction as $\mathcal{O}\left(\alpha^{3} \ln ^{3}\left(|t| / m_{e}^{2}\right)\right) / \ln \left(\bar{t} / m_{e}^{2}\right)$. For $\operatorname{ILC}_{500}$ it gives $0.028 \% / 20 \simeq 0.14 \times 10^{-4}$, in agreement with the former estimate.

\subsection{The $\gamma \otimes Z$ interferences}

At Born level there are a number of interference terms between $\gamma$ and $Z$ in $s$ and $t$-channel. The dominant contributions are, of course, the ones with t-channel photons. In BHLUMI 4.04 [3] only the $\left(Z_{s}+\gamma_{s}\right) \otimes \gamma_{t}$ are included. At LEP this approximation is accurate to $0.015 \%$, and it was acceptable.

This error can be reduced in the future because a complete calculation of the Bhabha matrix element up to the $\mathcal{O}(\alpha)$ is implemented in another Monte Carlo program: BHWIDE [29]. BHWIDE is also a YFS-based program with exponentiated photons to all orders.

The error due to the missing higher orders in BHWIDE was estimated in [1] as follows. The two sources of the error (added linearly) are: higher order QED corrections (nonsoft, i.e. not accounted for by the exponentiation) and higher order EW ones, beyond the $\mathcal{O}(\alpha)$. The QED non-soft h.o. terms have been estimated as $(\alpha / \pi) \ln \left(\bar{t}_{F C C e M_{Z}} / m_{e}^{2}\right)$ times the soft corrections calculated with BHWIDE i.e. $0.081 \times$ $0.042 \times 2 \simeq 0.007 \%$ (safety factor 2 included). The EW h.o. terms have been estimated in a similar way to be $0.003 \%$.
At ILC at $500 \mathrm{GeV}$ the $Z_{s}$ is far from resonance, so the dominant at LEP contribution $\gamma_{t} \otimes Z_{S}$ is no more enhanced by $M_{Z} / \Gamma_{Z}$ with respect to the $\gamma_{t} \otimes \gamma_{s}$ one. On the other hand the $\bar{t} / s$ is twice bigger than at LEP. This translates into similar relative enhancement of the $\gamma_{s}$-based interferences. The biggest change happens for $\bar{t}$ : from $2.9 \mathrm{GeV}^{2}$ at LEP to $149 \mathrm{GeV}^{2}$ at ILC, i.e. factor of 51. This increase will affect the $\gamma_{t} \otimes Z_{t}$ term.

The error components estimated for LEP in [1] are recalculated for ILC as follows:

1. $\gamma_{t} \otimes \gamma_{s}$ Born-level contribution gets suppressed by $\bar{t} / s$ with respect to $\gamma_{t} \otimes \gamma_{t}$ one, i.e. by $\sim 6 \times 10^{-4}$. The missing QED higher orders (beyond ones included in exponentiation) have been estimated in [1] as $10 \%$ of the Born-level contribution at $Z$ pole. At ILC these QED higher orders can increase from $10 \%$ by $\ln \left(\bar{t}_{I L C} / m_{e}^{2}\right) / \ln \left(\bar{t}_{L E P} / m_{e}^{2}\right) \simeq$ 1.24 to $13 \%$ of the Born-level contribution.

2. $\gamma_{t} \otimes Z_{s}$ Born-level contribution gets suppressed by factor of $1 / 4$ (due to coupling constant) with respect to $\gamma_{t} \otimes \gamma_{s}$ one . The higher orders we estimate by analogy with the $\gamma_{t} \otimes \gamma_{s}$ also as $13 \%$ of the Born-level contribution.

3. $\gamma_{t} \otimes Z_{t}$ Born-level contribution is suppressed with respect to $\gamma_{t} \otimes \gamma_{t}$ one by the factor $(1 / 4) \times \bar{t}_{I L C_{500}} / \mid \bar{t}_{I L C_{500}}+$ $M_{Z}^{2} \mid \simeq 0.0046$. Higher order terms we estimate again as $13 \%$ of the Born-level contribution, which is more conservative than $2 \times(\alpha / \pi) \ln \left(\bar{t}_{I L C_{500}} / m_{e}^{2}\right) \simeq 0.1$. As mentioned above, this huge increase w.r.t. LEP is due to change of $\bar{t}$.

4. $Z_{t} \otimes Z_{t}$ Born-level contribution is suppressed by another factor $(1 / 4) \times \bar{t}_{I L C_{500}} /\left|\bar{t}_{I L C_{500}}+M_{Z}^{2}\right| \simeq 0.0046$ as compared to $\gamma_{t} \otimes Z_{t}$ one i.e. it is of the order of $0.2 \times 10^{-4}$. Higher orders are negligible.

5. $\gamma_{s} \otimes \gamma_{s}$ Born-level contribution (and $Z_{s} \otimes Z_{s}$ Born-level contribution ) gets suppressed by another factor of $\bar{t} / s$ with respect to $\gamma_{t} \otimes \gamma_{s}$ one and is completely negligible.

At present only the items 1 and 2 are included in BHLUMI. Therefore the current BHLUMI error due to interferences for the ILC setup is high, around $0.5 \%$, almost entirely due to $\gamma_{t} \otimes Z_{t}$.

Assuming that the lowest order interferences are included in BHLUMI we can estimate total missing higher orders in ILC setup. Adding in quadratures all the components yields $6 \times 10^{-4}$ for ILC, again dominated by $\gamma_{t} \otimes Z_{t}$ Born-level contribution. Contributions of this size must be included in BHLUMI in a similar way as discussed for FCCee in [1], based on the complete $\mathcal{O}(\alpha)$ matrix element of the BHWIDE [29] type.

Now we need to do the estimate of missing $\mathcal{O}\left(\alpha_{E W}^{2}\right)$ terms at the higher energy of $500 \mathrm{GeV}$. In Ref. [30] the authors discussed briefly higher order corrections to the $\mathcal{O}(\alpha)$ EW corrections generated by the DIZET library [31] of ZFITTER 
[32]. The conclusion was that higher order corrections are estimated to be $0.025 \%$ at $800 \mathrm{GeV}$. Since these corrections grow with energy, we could safely use the same estimate at $500 \mathrm{GeV}$. However, more details on these corrections are given in an unpublished seminar presentation [33] by one of the authors of [30]. In the last figure on page 10 one finds there the actual size of these higher order corrections at 800 $\mathrm{GeV}$ to be below $0.005 \%$ for ILC angles. Applying safety factor of 2 we assume these corrections to be below $0.01 \%$ at ILC, in agreement with the previous estimate based on BHWIDE at $Z$ pole. The estimate was done in $[30,33]$ by changing switch $\operatorname{NPAR}(2)=3$ to $\operatorname{NPAR}(2)=4$.

\subsection{QED up-down interferences}

The interference between photonic emission from electron and positron lines is not included in BHLUMI. It is of the relative size of $0.07 \times \bar{t} / s$ [13]. At LEP this estimate gives a negligible value of $2.1 \times 10^{-5}$ in agreement with $1.4 \times 10^{-5}$ calculated in [13]. At ILC $\bar{t} / s$ grows by the factor of 2 and the correction is still on the verge of being negligible, below $4 \times$ $10^{-5}$. Inclusion of these interferences in the matrix element of BHLUMI does not pose any problems. The higher order contributions will be suppressed by an additional factor of $(\alpha / \pi) \ln \left(\bar{t} / m_{e}^{2}\right)$ and completely negligible for both setups.

\subsection{Vacuum polarisation}

Correction to $\mathrm{LABH}$ scattering due to the precision of the running coupling is proportional to $\delta \alpha(t)$ in the form $\delta \sigma_{B h} / \sigma_{B h}=2 \delta \alpha(t) / \alpha(t)$ where

$$
\begin{aligned}
\alpha(t)= & \frac{\alpha}{1-\Delta \alpha(t)}, \quad \Delta \alpha(t)=\Delta \alpha_{l e p}(t) \\
& +\Delta \alpha_{\text {had }}^{(5)}(t)+\Delta \alpha_{\text {top }}(t) .
\end{aligned}
$$

The error $\delta \alpha(t)$ is dominated by the error to the hadronic contribution $\Delta \alpha_{\text {had }}^{(5)}$ of five quarks. The errors from leptons and the top quark are negligible. $\delta \alpha(t)$ depends on the value of $t$. In Ref. [1] the authors discussed briefly this correction

for transfer $\bar{t}=12.5 \mathrm{GeV}$ which corresponds to FCCee setup at $350 \mathrm{GeV}$. Their conclusion was that $\delta \sigma_{B h} / \sigma_{B h} \simeq$ $2.4 \times 10^{-4}$ at present, with the future prospect of $1.2 \times 10^{-4}$ by the FCCee times.

Transfer $\bar{t}=12.5 \mathrm{GeV}$ also corresponds to the ILC setup at $500 \mathrm{GeV}$, which is the target of this work. We could, therefore, use directly the results of Ref. [1]. However, the more recent results on the hadronic vacuum polarisation can be found in Ref. [34]. In fig. B.1.15 of Ref. [34] a relative hadronic uncertainty $\delta \Delta \alpha_{\text {had }}(\sqrt{|t|})$ is presented as a function of $\sqrt{|t|}$. One can find there that at $-2 \mathrm{GeV}$ the uncertainty $\delta \Delta \alpha_{\text {had }}$ equals $0.8 \%$ of $\Delta \alpha_{\text {had }}$ whereas at $-12.5 \mathrm{GeV}$ it drops to $0.65 \%$. Together with the $\Delta \alpha_{\text {had }}(\sqrt{|t|})$ depicted in Fig. 2 of Ref. [35], i.e. $\Delta \alpha_{\text {had }}(-12.5)=0.016$, $\alpha(-12.5)=1 / 132$ and

$\frac{\delta \alpha(t)}{\alpha(t)}=\delta \Delta \alpha(t) \frac{\alpha(t)}{\alpha}$

we find out that $\delta \alpha(-12.5 \mathrm{GeV}) / \alpha(-12.5 \mathrm{GeV})=1.1 \times$ $10^{-4}$ and $\delta \sigma_{B h} / \sigma_{B h}=2.2 \times 10^{-4}$. This is consistent with the slightly older estimates of Ref. [1] quoted above. Following Ref. [1], as a future forecast we take half of the above error.

\subsection{Light pairs}

Emission of additional fermion pairs is not included in BHLUMI 4.04. The theoretical calculations of pair effect at the LEP energies were calculated by a few independent groups. First, semianalytical, calculations were based on LO and LO+NLO approximations, presented in Refs. [36,37]. The conclusion of Ref. [37] was that the precision of their calculation was $0.6 \times 10^{-4}$. Reference [36] was less optimistic and claimed precision to be $45 \%$ of the actual LO pair correction. These results were obtained with not fully realistic, simplified, acceptances. The approach of Ref. [38] was based on a four-fermion Monte Carlo and realistic acceptances. It claimed precision of $1 \times 10^{-4}$. Finally, in Refs. [39,40] yet another approach was taken. It was based on extension of YFS soft photon resummation to the emission of soft pairs and implemented in an unpublished version of BHLUMI 2.30. The precision was established at the level of $2 \times 10^{-4}$. Numerical results were given in Refs. [39,40] only for simplified acceptances.

The most recent analysis of pair emission in $\mathrm{LABH}$ at LEP was given in Ref. [7]. The methodology extends the above two analyses in the spirit of the 1994 ALEPH analysis [41]. Real emissions were simulated with the dedicated MC code FERMISV [42] with all $\gamma$ and $Z$ exchange fourfermion graphs supplied with LL ISR and FSR. FERMISV was augmented in very small angles by the four-fermion code KoralW $[43,44]$. Simulations included all fermion species. The quark pairs were corrected with the R-ratio to emulate hadronic final states. The virtual corrections were taken from Ref. [26]. The error estimate in Ref. [7] was based on a comparison with the results from Ref. [38]. The comparison showed agreement at the level of $0.2 \times 10^{-4}$. This number was increased by factor of 3 to account for the limitations of FERMISV and KoralW very close to the beam pipe. The missing higher order corrections were estimated as the $20 \%$ of the entire correction, i.e. $0.8 \times 10^{-4}$. These two errors combined gave final error of $1 \times 10^{-4}$.

Having outlined the state of the art in light pair contribution let us discuss future prospects of reducing this error. We will present two ways: adding to BHLUMI 4.04 upgraded pair emissions a la YFS or, following Ref. [7], use the dedicated four fermion generator. In the following we use LEP setup. 
(1) External four-fermion code. A weak point of the available four-fermion codes is the photonic radiation based on the $s$-channel oriented ISR. The LABH kinematics is $t$ channel oriented and so should be the bremsstrahlung. Naive comparison of the size of $\ln \left(\bar{t}_{L E P} / m_{e}^{2}\right)$ and $\ln \left(s_{M_{Z}} / m_{e}^{2}\right)$ shows a $30 \%$ difference. We take that $30 \%$ as the precision of the $s$-channel oriented ISR. In Ref. [7] the pair corrections with the full detector acceptances were calculated to be at the level of $4 \times 10^{-4}$. In Refs. [37,38] it was shown that the single-photon correction can be as big as 20 $30 \%$ for the simplified acceptances. These three above numbers combined yield the single-photon correction to be of the order of $25 \% \times 4 \times 10^{-4}=1 \times 10^{-4}$ with the error $30 \% \times 1 \times 10^{-4}=0.3 \times 10^{-4}$. The non-leading photonic corrections are suppressed by $1 / \ln \left(\bar{t}_{L E P} / m_{e}^{2}\right)$ and negligible. The next sources of error, higher order photonic $(2 \gamma)$ corrections and emission of a second pair, are suppressed by $(\alpha / \pi) \ln \left(\bar{t}_{L E P} / m_{e}^{2}\right)$ and $\left((\alpha / \pi) \ln \left(\bar{t}_{L E P} / m_{e}^{2}\right)\right)^{2}$ respectively, i.e. negligible even when a safety factor of 2 is included (below $0.1 \times 10^{-4}$ ). Another question is the accuracy of the description of the hadronic emissions based on the R-ratio of hadronic to muonic cross-section in $e^{+} e^{-}$annihilation. According to Ref. [41] this accuracy is of the order of a few per cent, hence negligible (below $0.1 \times 10^{-4}$ ). The technical precision related to the limitations of codes close to the beam pipe can be reduced either by choosing one of the other available 4-fermion codes, or, in the case of Kora 1W, by using the dedicated quadruple precision version. This way the technical precision of $5 \%$, i.e. $0.2 \times 10^{-4}$, should be easily achieved. The above analysis shows that the total error of this approach can be $0.4 \times 10^{-4}$. This number can be further reduced if a four-fermion code with the complete $\mathcal{O}(\alpha)$ corrections [45] becomes available for all the decay channels and the $\mathrm{LABH}$ kinematics. In such a case, the uncertainty of the photonic radiation would drop significantly.

(2) Upgrade of BHLUMI with $t$-channel oriented ISR. The real power of this approach are the $t$-channel based photonic corrections implemented in BHLUMI. These corrections are resummed in the soft limit and supplemented to complete $\mathcal{O}\left(\alpha^{2} \ln ^{2}\left(\bar{t} / m_{e}^{2}\right)\right)$ LO ISR, thus, contrary to scenario (1), they do not contribute to the error budget (non-leading non-soft corrections are suppressed by $\left.1 / \ln \left(\bar{t}_{L E P} / m_{e}^{2}\right)\right)$. The item that should be upgraded in BHLUMI is the four-fermion matrix element. In the $4 e$ channel it misses the multiperipheral graphs. While negligible in the soft pairs limit, these graphs contribute up to $15 \%$ for $z=0.5$ and $30 \%$ for $z=0.7$ (hard emission) [38]. It is easy to fix with modern matrix element generators. Two other leptonic channels, $2 e 2 \mu$ and $2 e 2 \tau$ must be added in a similar manner. As before, the accuracy of these matrix elements should easily reach the level of $0.2 \times 10^{-4}$. The quark pairs can be added as in the previous case with the help of the $R$-ratio and precision below $0.1 \times 10^{-4}$. The $\mathcal{O}(\alpha)$ four-fermion code most likely will not be necessary. Summarising, the accuracy of this approach could be $0.2 \times 10^{-4}$.

The above discussion, extending analysis from Ref. [1], was done for the LEP setup. Now let us discuss what will happen in the case of ILC. The LABH cross section is governed by the transfer $t$ rather than $s$ and the resonant structure of the $s$-channel $Z$-boson. The $\ln \left(\bar{t}_{I L C} / m_{e}^{2}\right)$ grows from 16.26 at LEP to 20.2 , i.e. by a factor 1.24 (photonic emission). The square of that logarithm, responsible for pair emission, grows by factor 1.54 .

Applying these numbers to scenario (1) we find that the pair correction will grow up to $1.54 \times 4 \times 10^{-4}=6 \times 10^{-4}$. A more refined analysis can be done by splitting the $4 \times$ $10^{-4}$ LEP pair correction into $e$-pairs $\left(3.3 \times 10^{-4}\right)$ and $\mu+$ hadr-pairs $\left(0.7 \times 10^{-4}\right)$ according to the appropriate ratios of logarithms, as described in [1], and then evolving $e$-pair component with $\bar{t}$ and reconstructing $\mu+$ hadr component at higher $\bar{t}$. This procedure gives $8 \times 10^{-4}$ instead of $6 \times 10^{-4}$. Pair plus photon correction will increase to $25 \% \times 8 \times 10^{-4}=$ $2 \times 10^{-4}$ with the error $36 \% \times 2 \times 10^{-4}=0.7 \times 10^{-4}$, where we exploited the change $\ln \left(\bar{t}_{I L C} / m_{e}^{2}\right)$ vs. $\ln s_{500} / m_{e}^{2} \sim 36 \%$. The non-leading photonic corrections will be around $2 \times$ $10^{-4} / \ln \left(\bar{t}_{I L C} / m_{e}^{2}\right) \simeq 0.1 \times 10^{-4}$. The missing higher order corrections $(2 \gamma)$ will increase to $0.2 \times 10^{-4}$ (with safety factor of 2). Precision of hadronic correction remains below $0.1 \times 10^{-4}$ and the technical precision of the four-fermion codes is estimated as for LEP at $5 \% \times 8 \times 10^{-4}=0.4 \times$ $10^{-4}$. In total we obtain at ILC the precision $0.8 \times 10^{-4}$. As before, that number can be significantly reduced if the $\mathcal{O}(\alpha)$ four fermion code becomes available, perhaps to $0.5 \times 10^{-4}$ (assuming $10 \% \times 2 \times 10^{-4}=0.2 \times 10^{-4}$ as precision of the $\mathcal{O}(\alpha)$ correction).

In the case of scenario (2) the analysis of the matrix element upgrade remains unchanged and yields a technical error of $0.4 \times 10^{-4}$, as in the case (1). The non-leading photonic emission uncertainty is $0.1 \times 10^{-4}$, the same as in the scenario (1); so is the precision of the hadronic corrections. The total precision of scenario (2) we estimate to be $0.4 \times 10^{-4}$ at ILC.

It is remarkable that scenario with the upgrade of BHLUMI is more precise thanks to a better treatment of the photonic radiation.

\section{Technical precision of BHLUMI}

So far, we have discussed only the physical errors, due to the missing corrections. The only exception has been done for the case of the four-fermion matrix elements. In that case, the specific stability issues arise in the relevant for us limit of electrons very close to the beam pipe. We added these errors to the physical ones. 
Table 3 Forecasts of the total (physical+technical) theoretical uncertainty for the ILC 500 luminosity calorimetric detector with the angular range of 31-77 mrad. Description of photonic corrections in square brackets is related to the 2 nd column. The numbers in curly brackets in lines (e) and "Total" refer to a fictitious situation with all Born-level interferences included in BHLUMI. The total error is summed in quadrature. A technical error is not included in "Total"

\begin{tabular}{lll}
\hline ILC 500 setup & & \\
\hline Type of correction/error & Update 2019 & Forecast \\
\hline (a) Photonic $\left[\mathcal{O}\left(L_{e} \alpha^{2}\right)\right] \mathcal{O}\left(L_{e}^{2} \alpha^{3}\right)$ & $0.033 \%$ & $0.13 \times 10^{-4}$ \\
(b) Photonic $\left[\mathcal{O}\left(L_{e}^{3} \alpha^{3}\right)\right] \mathcal{O}\left(L_{e}^{4} \alpha^{4}\right)$ & $0.028 \%$ & $0.27 \times 10^{-4}$ \\
(c) Vacuum polariz. & $0.022 \%[34]$ & $1.1 \times 10^{-4}$ \\
(d) Light pairs & $0.010 \%[7]$ & $0.4 \times 10^{-4}$ \\
(e) $Z$ and $s$-channel $\gamma$ exchange & $0.5 \%(0.06 \%)$ & $1.0 \times 10^{-4}$ \\
(f) Up-down interference & $0.004 \%[13]$ & $<0.1 \times 10^{-4}$ \\
(g) Technical Precision & $(0.027 \%)$ & $0.1 \times 10^{-4}$ \\
Total & $0.5 \%(0.078 \%)$ & $1.6 \times 10^{-4}$ \\
\hline
\end{tabular}

The technical precision of the BHLUMI code is a separate issue. As discussed in Ref. [1], the ultimate estimate comes from a comparison with another MC code, based on a different approach and implementation. In the case of LEP this method was somewhat limited and yielded the number of $0.027 \%$. It resulted not only from the comparison with different codes (SABSPV, LUMLOG, OLDBIS) but also with tedious dedicated semi-analytical calculations. In the future we hope that other Monte Carlo codes will appear and high accuracy comparisons will be possible. As an example we can mention an interesting code BabaYaga [46-48] developed at the end of the LEP era. BabaYaga also uses softphoton resummation, however based on the parton shower method.

Another foreseeable approach could be to develop a new version of BHLUMI based on a more sophisticated exponentiation done at the level of spin amplitudes. This is contrary to the standard YFS-based approach done with the squared amplitudes.

To summarise, at the moment one cannot give a solid number for the future technical precision. We put into the Table 3 the number $0.001 \%$ more as an expectation and do not combine it with the physical precision.

\section{Summary}

In this note we have discussed a possible precision of the $\mathrm{LABH}$ calculation used for the luminosity measurement. As a starting point we have used the similar analysis done for the FCCee experiment in Ref. [1]. Our findings for the ILC are collected in Table 3. In the second column, we show the current situation with the ILC $_{500}$ setup and the third column gives the $\mathrm{ILC}_{500}$ forecast.

The current total precision of $(0.5 \%)$ is so low due to the trivial $\gamma_{t} \otimes Z_{t}$ interference missing in BHLUMI. Assuming that this is taken care of, the precision of interferences drops down to $0.06 \%$ and the total error to $0.078 \%$ (numbers shown in the curly brackets in the Table 3 ).

As one can see, the forecasted final precision is worse by the factor of nearly two as compared to the FCCee $_{M_{Z}}$ forecast. The dominant error component is still the hadronic vacuum polarisation, which grows by the factor of two when moving from $\mathrm{FCCee}_{M_{Z}}$ to ILC transfer $t$. The second largest component are the $\mathcal{O}\left(\alpha^{2}\right) \mathrm{EW}$ and ISR corrections. They are estimated based on the assumption that the matrix element of BHWIDE type is implemented in BHLUMI. This uncertainty is only slightly bigger than at $\mathrm{FCCee}_{M_{Z}}$.

At last, in Table 4 we present the forecast for other planned experiments: $\mathrm{FCCe}_{350}, \mathrm{ILC}_{1000}$ and CLIC for the setups as defined in Table 1. These results are obtained with the same methodology as described in the paper. The striking feature of Table 4 is a complete dominance at higher energies of the error due to missing unknown $\mathrm{EW} \mathcal{O}\left(\alpha^{2}\right)$ corrections, line (e); that estimate is based on Ref. [30].

Data Availability Statement This manuscript has no associated data or the data will not be deposited. [Authors' comment: This is a purely theoretical paper with no data to deposit.]

Open Access This article is licensed under a Creative Commons Attribution 4.0 International License, which permits use, sharing, adaptation,
Table 4 Forecast of the total (physical) theoretical uncertainty for the $\mathrm{FCCee}_{350}$, ILC $_{1000}$ and CLIC luminosity calorimetric detectors with angular acceptances as defined in the text. Number $\left(^{\star}\right)$ is likely overestimated. The total error is summed in quadrature. A technical error is not included

\begin{tabular}{llll}
\hline Forecast & & & \\
\hline Type of correction/error & FCCee $_{350}$ & ILC $_{1000}$ & CLIC $_{3000}$ \\
\hline (a) Photonic $\mathcal{O}\left(L_{e}^{2} \alpha^{3}\right)$ & $0.13 \times 10^{-4}$ & $0.15 \times 10^{-4}$ & $0.20 \times 10^{-4}$ \\
(b) Photonic $\mathcal{O}\left(L_{e}^{4} \alpha^{4}\right)$ & $0.27 \times 10^{-4}$ & $0.37 \times 10^{-4}$ & $0.63 \times 10^{-4}$ \\
(c) Vacuum polariz. & $1.1 \times 10^{-4}$ & $1.1 \times 10^{-4}$ & $1.2 \times 10^{-4}$ \\
(d) Light pairs & $0.4 \times 10^{-4}$ & $0.5 \times 10^{-4}$ & $0.7 \times 10^{-4}$ \\
(e) $Z$ and $s$-channel $\gamma$ exchange & $1.0 \times 10^{-4(*)}$ & $2.4 \times 10^{-4}$ & $16 \times 10^{-4}$ \\
(f) Up-down interference & $0.1 \times 10^{-4}$ & $<0.1 \times 10^{-4}$ & $0.1 \times 10^{-4}$ \\
Total & $1.6 \times 10^{-4}$ & $2.7 \times 10^{-4}$ & $16 \times 10^{-4}$ \\
\hline
\end{tabular}


distribution and reproduction in any medium or format, as long as you give appropriate credit to the original author(s) and the source, provide a link to the Creative Commons licence, and indicate if changes were made. The images or other third party material in this article are included in the article's Creative Commons licence, unless indicated otherwise in a credit line to the material. If material is not included in the article's Creative Commons licence and your intended use is not permitted by statutory regulation or exceeds the permitted use, you will need to obtain permission directly from the copyright holder. To view a copy of this licence, visit http://creativecomm ons.org/licenses/by/4.0/.

Funded by SCOAP ${ }^{3}$.

\section{References}

1. S. Jadach, W. Płaczek, M. Skrzypek, B.F.L. Ward, S.A. Yost, Phys. Lett. B 790, 314-321 (2019). arXiv:1812.01004

2. S. Jadach, E. Richter-Was, B.F.L. Ward, Z. Was, Comput. Phys. Commun. 70, 305-344 (1992)

3. S. Jadach, W. Placzek, E. Richter-Was, B.F.L. Ward, Z. Was, Comput. Phys. Commun. 102, 229-251 (1997)

4. M. Dam, arXiv:2107.12837

5. FCAL Collaboration, V. Ghenescu, W. Lohmann, PoS EPS-HEP 2019, $210(2020)$

6. A.C. Abusleme Hoffman et al., CERN Yellow Rep. Monogr (2019). https://doi.org/10.23731/CYRM-2019-001. arXiv:1905.02520

7. P. Janot, S. Jadach, Phys. Lett. B 803, 135319 (2020). arXiv:1912.02067

8. S. Jadach, M. Melles, B.F.L. Ward, S.A. Yost, Acta Phys. Pol. B 30, 1745-1750 (1999)

9. S. Jadach, B.F.L. Ward, Phys. Lett. B 389, 129-136 (1996)

10. F. Jegerlehner, in FCC-ee Mini-Workshop, "Physics Behind Precision" (2016). https://indico.cern.ch/event/469561/contributions/ 1977974/attachments/1221704/1786449/SMalphaFCCee16.pdf

11. S. Jadach, W. Placzek, B.F.L. Ward, Phys. Lett. B 353, 349-361 (1995)

12. A. Arbuzov et al., Phys. Lett. B 383, 238-242 (1996). arXiv:hep-ph/9605239

13. S. Jadach, E. Richter-Was, B.F.L. Ward, Z. Was, Phys. Lett. B 253, 469-477 (1991)

14. D.R. Yennie, S.C. Frautschi, H. Suura, Ann. Phys. 13, 379-452 (1961)

15. S. Jadach, Yennie-Frautschi-Suura Soft Photons in Monte Carlo Event Generators. MPI-PAE/PTh 6/87, 1 (1987)

16. R. Kleiss, W.J. Stirling, Nucl. Phys. B 262, 235-262 (1985)

17. CALKUL Collaboration, F.A. Berends, P. De Causmaecker, R. Gastmans, R. Kleiss, W. Troost, T.T. Wu, Nucl. Phys. B 264, 265-276 (1986)

18. S. Jadach, M. Melles, B.F.L. Ward, S.A. Yost, Phys. Lett. B 377, 168-176 (1996). arXiv:hep-ph/9603248

19. F.A. Berends, W.L. van Neerven, G.J.H. Burgers, Nucl. Phys. B 297, 429 (1988) [Erratum: Nucl. Phys. B 304, 921 (1988)]

20. S. Jadach, B.F.L. Ward, Acta Phys. Pol. B 28, 1907-1979 (1997)

21. M. Czakon, J. Gluza, T. Riemann, Acta Phys. Pol. B 36, 3319-3326 (2005). arXiv:hep-ph/0511187

22. A.A. Penin, Nucl. Phys. B 734, 185-202 (2006). arXiv:hep-ph/0508127
23. Z. Bern, L.J. Dixon, A. Ghinculov, Phys. Rev. D 63, 053007 (2001). arXiv:hep-ph/0010075

24. R. Bonciani, A. Ferroglia, Phys. Rev. D 72, 056004 (2005). arXiv:hep-ph/0507047

25. S. Actis, M. Czakon, J. Gluza, T. Riemann, Nucl. Phys. B 786, 26-51 (2007). arXiv:0704.2400

26. S. Actis, M. Czakon, J. Gluza, T. Riemann, Phys. Rev. D 78, 085019 (2008). arXiv:0807.4691

27. R. Bonciani, A. Ferroglia, A.A. Penin, Phys. Rev. Lett. 100, 131601 (2008). arXiv:0710.4775

28. J.H. Kuhn, S. Uccirati, Nucl. Phys. B 806, 300-326 (2009). arXiv:0807.1284

29. S. Jadach, W. Placzek, B.F.L. Ward, Phys. Lett. B 390, 298-308 (1997). arXiv:hep-ph/9608412

30. M. Battaglia, S. Jadach, D. Bardin, eConf C010630, E3015 (2001). http://www.slac.stanford.edu/econf/C010630/papers/E3015.PDF

31. DYu. Bardin, M.S. Bilenky, T. Riemann, M. Sachwitz, H. Vogt, Comput. Phys. Commun. 59, 303-312 (1990)

32. D.Y. Bardin, P. Christova, M. Jack, L. Kalinovskaya, A. Olchevski, S. Riemann, T. Riemann, Comput. Phys. Commun. 133, 229-395 (2001). arXiv:hep-ph/9908433

33. S. Jadach, MC tools for extracting luminosity spectra. What do we need? (2002). https://jadach.web.cern.ch/jadach/public/ LumLCslac.pdf

34. F. Jegerlehner, CERN Yellow Rep. Monogr. 3, 9-37 (2020)

35. F. Jegerlehner, Nucl. Phys. Proc. Suppl. 162, 22-32 (2006). arXiv:hep-ph/0608329

36. S. Jadach, M. Skrzypek, B.F.L. Ward, Phys. Rev. D 47, 3733-3741 (1993)

37. A.B. Arbuzov, V.S. Fadin, E.A. Kuraev, L.N. Lipatov, N.P. Merenkov, L. Trentadue, Nucl. Phys. B 485, 457-502 (1997). arXiv:hep-ph/9512344

38. G. Montagna, M. Moretti, O. Nicrosini, A. Pallavicini, F. Piccinini, Nucl. Phys. B 547, 39-59 (1999). arXiv:hep-ph/9811436

39. S. Jadach, M. Skrzypek, B.F.L. Ward, Phys. Rev. D 49, 1178-1182 (1994)

40. S. Jadach, M. Skrzypek, B.F.L. Ward, Phys. Rev. D 55, 1206-1215 (1997)

41. ALEPH Collaboration, D. Buskulic et al., Z. Phys. C 66, 3-18 (1995)

42. J. Hilgart, R. Kleiss, F. Le Diberder, Comput. Phys. Commun. 75, 191-218 (1993)

43. S. Jadach, W. Placzek, M. Skrzypek, B.F.L. Ward, Z. Was, Comput. Phys. Commun. 119, 272-311 (1999). arXiv:hep-ph/9906277

44. S. Jadach, W. Placzek, M. Skrzypek, B.F.L. Ward, Z. Was, Eur. Phys. J. C 27, 19-32 (2003). arXiv:hep-ph/0209268

45. A. Denner, S. Dittmaier, M. Roth, L.H. Wieders, Nucl. Phys. B 724, 247-294 (2005). arXiv:hep-ph/0505042 [Erratum: Nucl. Phys. B 854, 504-507 (2012)]

46. C.M. Carloni Calame, C. Lunardini, G. Montagna, O. Nicrosini, F. Piccinini, Nucl. Phys. B 584, 459-479 (2000). arXiv:hep-ph/0003268

47. C.M. Carloni Calame, Phys. Lett. B 520, 16-24 (2001). arXiv:hep-ph/0103117

48. G. Balossini, C.M. Carloni Calame, G. Montagna, O. Nicrosini, F. Piccinini, Nucl. Phys. B 758, 227-253 (2006). arXiv:hep-ph/0607181 